\title{
THE CHALLENGING PARADIGM OF PHARMACEUTICAL CARE
}

\author{
Ivo Kumanov \\ Faculty of Pharmacy, Department of Pharmaceutical Sciences \\ and Pharmaceutical Management, Medical University of Varna
}

\begin{abstract}
Pharmaceutical care is designed to aid medical professionals in ensuring optimal patient's management. The necessity of elaboration and practical implementation of an appropriate philosophy of pharmaceutical practice and its organizational structures was suggested 25 years ago by Charles D. Hepler and Linda M. Strand. They defined the terms of 'pharmaceutical care' and 'pharmaceutical-care system'. Pharmaceutical care provides drug therapy for achieving definite outcomes that improve a patient's quality of life, namely: cure of a disease; reduction or elimination of symptoms; arresting or slowing of a disease process as well as preventing a disease or symptoms. There is strong evidence of the feasibility and effectiveness of a variety of interventions immediately associated with pharmaceutical care aiming at improving patients' relationships with master pharmacists in the community and hospital pharmacies. The importance of interactive pharmacy students' learning for enhancement of their pharmaceutical care competences is emphasized. Hospital pharmacists recommend a broader practical implementation of pharmaceutical care while appreciating the organizational, technical and professional barriers in this respect. Collaborative efforts between health authorities and educational institutions along with the integration of innovative approaches in the management of public and hospital pharmacies and pharmacy education could contribute to overcome these barriers.
\end{abstract}

Keywords: pharmaceutical care paradigm, pharmacists, higher education, patients, attitudes, barriers

\section{INTRODUCTION}

In the USA, drug misadventuring is derived from the actions or inactions of a variety of social, economic, political, and scientific-technological 'environments' (1). Prescribers, patients, pharmacists, manufacturers and policy-makers directly influence

\footnotetext{
Address for correspondence:

Ivo Kumanov, MS

Department of Pharmaceutical Sciences and Pharmaceutical

Management

Faculty of Pharmacy

Medical University "Prof. Paraskev Stoyanov" of Varna

55 Marin Drinov Street

9002 Varna, Bulgaria

e-mail:ivo.kumanov@abv.bg
}

Received: Desember 1, 2015

Accepted: December 30, 2015 on decisions associated with drug misadventuring. This public policy problem necessitates the construction of new policy decisions in the best interest of the patient. In 1987, about 12000 deaths and 15000 hospitalizations due to adverse drug reactions have been reported to the Food and Drug Administration in the USA $(1,2)$. Drug-related morbidity and mortality are often preventable, and pharmaceutical services can reduce the number of adverse drug reactions, length of hospital stays, and cost of care.

Pharmacists must abandon factionalism and adopt patient-centered pharmaceutical care as their philosophy of practice (3). Changing the focus of practice from products and biological systems to ensuring the best drug therapy and patient safety raise pharmacy's level of responsibility and require philosophical, organizational, and functional changes. It is necessary to set new practice standards, estab- 
lish cooperative relationships with other health-care professions, and determine strategies for marketing pharmaceutical care. Pharmacy's reprofessionalization will be completed only when all pharmacists accept their social mandate to ensure the safe and effective drug therapy of the individual patient.

\section{ORIGIN OF PHARMACEUTICAL CARE PARADIGM}

Pharmacy's societal function should involve 'pharmaceutical care', a concept that includes both drug-product control and clinical pharmacy services (4). There must be an appropriate philosophy of practice and an organizational structure within which to practice. In 1990, pharmaceutic care has been suggested as paradigm of health for all in the year 2000 (5).

C. D. Hepler and L. M. Strand (1990) comprehensively explore pharmacy's opportunity to mature as a profession by accepting its social responsibility to reduce preventable drug-related morbidity and mortality. Pharmacy has shed the apothecary role but has not yet been restored to its erstwhile importance in medical care. It is neither enough to dispense the correct drug or to provide sophisticated pharmaceutical services, nor to devise new technical functions. Pharmacists and their institutions must stop looking inward and start redirecting their energies to the greater social good.

Practitioners of pharmaceutical care are concerned with the effect of their services on patients' quality of life and not merely with the act of providing services (6). They work with other health-care professionals as equals to ensure that therapeutic goals are achieved and that drug-related illness does not occur or is quickly detected and resolved. To be accepted and implemented, pharmaceutical care must first overcome pharmacy's product- and service-oriented focus, opposition from other healthcare professions and drug manufacturers, financial and logistical problems, and ignorance and inertia among pharmacists themselves. Through a united effort, pharmacy organizations, schools and individual pharmacists can translate the need for pharmaceutical care into demands for it by patients, insurance companies, health maintenance organizations and the government as well.
Drugs are administered for the purpose of achieving definite outcomes that improve patient's quality of life $(3,7)$. These outcomes are the following: 1) cure of a disease; 2 ) reduction or elimination of symptoms; 3) arresting or slowing of a disease process, and 4) preventing a disease or symptoms. Pharmaceutical care is the responsible provision of such a drug therapy and involves three major functions on behalf of the patients: 1) identifying potential and actual drug-related problems; 2 ) resolving actual drugrelated problems, and 3) preventing potential drugrelated problems. Through the process of pharmaceutical care a pharmacist cooperates with a patient and other professionals in designing, implementing and monitoring a therapeutic plan that will produce specific therapeutic outcomes for the patient.

It represents a necessary element of health care and should be integrated with other elements. The pharmacist is responsible directly to the patient for the quality of this care. The fundamental relationship in pharmaceutical care is a mutually beneficial exchange in which the patient grants authority to the provider and the provider promises competence and commitment (accepts responsibility) to the patient.

C. D. Hepler and L. M. Strand (1990) term the necessary philosophy of practice 'pharmaceutical care' and the organizational structure that facilitates the provision of this care the 'pharmaceutical caresystem'. The mission of the pharmaceutical practice is to provide pharmaceutical care (4).

Meanwhile, growing numbers of practitioners worldwide have adopted pharmaceutical care as an integral component of pharmacy practice. Established models of pharmaceutical care can reduce the burden of preventable drug therapy problems. Although many challenges remain, the profession has key resources to expand and improve the delivery of pharmaceutical care (8).

PHARMACISTS' ATTITUDES TOWARDS PHARMACEUTICAL CARE

The pharmacists begin to accept the mandate of pharmaceutical care when there is widespread training and use of technicians, implementation of problem-based education in the schools, and adequate problem-solving support for pharmacists in practice (9). 
The results from a cross-sectional survey among 86 Eritrean pharmacists indicate that $95 \%$ of them perceive that their current role needs to be redefined and re-oriented (10). Some $86 \%$ of them believe there is a great mismatch between pharmacists' levels of education and practice in the work area. Although they are proud to be pharmacy professionals, they want to redefine and reorient their current role into pharmaceutical care.

Within a descriptive, cross-sectional study of 250 pharmacists working in the governmental hospitals in Kuwait, overall positive attitudes towards pharmaceutical care are expressed (11). They feel well-prepared to implement the various aspects of pharmaceutical care, with the least preparedness in the administrative/management aspects. The pharmacists with more practice experience present with significantly more positive attitudes towards pharmaceutical care $(\mathrm{p}=0.001)$ and feet better prepared to provide pharmaceutical care competencies $(\mathrm{p}<0.001)$ than those with less practical experience. The respondents agree/strongly agree that the most significant barriers to the integration of pharmaceutical care into practice are lack of private counseling areas or inappropriate pharmacy layout $(87.6 \%)$, organizational obstacles $(81.6 \%)$, inadequate staff $(79.6 \%)$ as well as lack of pharmacist's time and adequate technology (76.0\% of the cases). Collaborative efforts between health authorities and educational institutions, and the integration of innovative approaches in pharmacy management and education could overcome these barriers and achieve the transition towards pharmaceutical care practice.

When ambulatory care pharmacists engage in pharmaceutical patient care to their full capacity, physician time is saved, access to care is improved as well as clinical and economic outcomes are enhanced (12). There is a need for ambulatory care pharmacists to work toward optimizing the safe medication use and drug therapy for patients with diabetes mellitus, bronchial asthma, cardiovascular and renal diseases. There exist other opportunities for the development of ambulatory care pharmacy services in preventive care, precision therapeutics, medication therapy management, mitigation of healthcare disparities, and implementation of national healthcare reform. Interprofessional patient care teams include ambulatory care pharmacists in patient-centered medical homes and accountable care organizations. The ambulatory care pharmacy practice benefits by enhancing the specialty residency training and by creating the residency/fellowship for advanced subspecialty clinical practice and research. By assertively such advancing practice, pharmacy can help achieve the national priorities of improving patient care, patient health, and affordability of care. Provider status is essential to recognize pharmacists as an integral part of the patient care team.

A cross-sectional study among 80 managing pharmacists in 125 randomly selected community pharmacies in Flanders, Belgium, is performed (13). Usage is made of structured interviews concerning the current implementation level of PHARMACOPprotocol that focuses on patient counselling within pharmaceutical care during prescription filling for chronic obstructive pulmonary disease. For first and follow-up prescriptions, $49 \%$ and $43 \%$ of pharmacists, respectively, need to spend at least five min extra to offer optimal patient counselling. Most barriers for protocol implementation include lack of time (in $80 \%$ ), no integration in pharmacy software (in $61 \%$ ), and too much administrative burden (in 58\% of the responses).

The analysis of 51 surveys completed by community pharmacists in New York, the USA, demonstrates that $90 \%$ of the respondents are confident in providing counselling about medications used to manage chronic kidney disease, $63 \%$ indicate confidence in recommending drug dosing changes based on kidney function, $88 \%$ indicate a willingness to play a greater role in reviewing medications for such patients, and all agree that they will benefit from education about the complications of this disease and their management (14). The enhancement of the collaborative pharmaceutical care for patients with chronic kidney disease is emphasized.

Based on a pilot study about pharmaceutical care implantation in a general paediatric unit in a French hospital it is concluded that French pharmacists can apply the pharmaceutical care model following a specific training in a patient unit of a French pediatric hospital (15). 
PATIENTS' ATTITUDES TOWARDS PHARMACEUTICAL CARE

Within a randomized controlled trial among 380 patients from a public emergency department at Restinga district in Porto Alegre, Southern Brazil, it has been established that pharmaceutical care interventions are feasible and effective in increasing the medication adherence in both hospital outpatient and community pharmacy settings (16).

The intervention consisting of clinical pharmacist medication reconciliation, patient education and improved communication of the discharge medication plan as devised by the hospital physician and care team to primary care physicians and community pharmacists and covering 592 patients from internal medicine, family medicine, cardiology and orthopedic services may affect the quality of the outpatient medical record of these patients expressed by smaller mean number of medication discrepancies per patient for the enhanced group than that for the control group (17).

Usage of patient's self-completion concordance forms by 99 patients in 24 Dutch and by 241 patients in 41 Bulgarian pharmacies is evaluated (18). A higher proportion of Bulgarian patients answer questions on these forms compared to Dutch ones. The patients from both countries are satisfied with the selfcompletion concordance forms, consultation and newly-started medicine. These forms can be used in community pharmacies at the start of chronic treatment and in order to develop indicators to measure patient's involvement in pharmaceutical care.

The results from a pragmatic randomised controlled trial of 144 women with epilepsy aged over 18 years demonstrate that the application of a pharmaceutical care programme significantly improves their health-related quality of life (19).

During a prospective study of renal transplant recipients, 35 patients receive the standardized drug and transplant training while 32 ones receive intensified additional inpatient and outpatient pharmaceutical care and counselling by a dedicated clinical pharmacist (20). There is a significant improvement of patients' immunosuppressive medication adherence during the first post-transplant year that suggests that this pharmaceutical care has the potential to improve outcomes after organ transplantation.
Within a randomized controlled study in China, the individualized pharmaceutical care exerts a statistically significant favorable effect on medication adherence at six-month $(\mathrm{p}=0.016)$ and at oneyear follow-up $(\mathrm{p}=0.039)$ as well as on hospital admission $(\mathrm{p}=0.01)$ in chronic obstructive pulmonary disease patients (21).

In a randomized controlled trial of 945 participants are assigned to minimal, enhanced and usual pharmaceutical care groups (22). The minimal group receives admission history, medication reconciliation, patient education, discharge medication list and medication recommendations to inpatient team and the enhanced one receives a faxed medication care plan to the community physician and pharmacy and telephone call three-five days post-discharge, too. The average medication appropriateness index per medication is 0.53 at discharge from hospital and increases up to 0.75 at 90 days, and this is true across all study groups.

STUDENT'S EDICATION IN PHARMACEU-

\section{TICAL CARE}

The performance and perceptions of competency of 33 students in a new pharmaceutical care course conducted in the first semester of 2014, in the Federal University of Sergipe, Brazil, that uses active learning methodologies are evaluated (23). The overall mean of students' scores on a scale of 0 to 10 points is $7.97 \pm 0.59$, as student's performance on the virtual patient method is statistically superior to other methods. With respect to the perception of competency in pharmaceutical care practice, a comparison of pre- and post-test scores reveals statistically significant improvement for all evaluated competences. Finally, the students present positive opinions of this pharmaceutical care course.

The results from a descriptive, cross-sectional survey of 126 pharmacy students in the Faculty of Pharmacy, Kuwait University, Kuwait, indicate their overall positive attitudes towards pharmaceutical care (24). They feel prepared to implement the various aspects of pharmaceutical care, with the least preparedness in the administrative/management aspects and appreciate the importance of the various pharmaceutical care competencies. The pharmacy students agree/strongly agree that the major barriers to the integration of pharmaceutical care into 
practice are lack of private counseling areas or inappropriate pharmacy layout (95.2\%), lack of pharmacists' time (83.3\%), organizational obstacles (82.6\%), and pharmacists' physical separation from patient care areas (82.6\% of the respondents). Their attitudes and perceived preparedness can serve as needs assessment tools to guide curricular change and improvement. This policy enables their development as change agents who can advance pharmacist-provided direct patient care.

The results from a questionnaire study of 143 final-year students in pharmacy preparing to become community pharmacists, at the University Paris-Descartes in Paris, France, indicate their attitudes towards the future provision of pharmaceutical care (25). The most significant barriers perceived by the students are the following: working time, remuneration and organizational problems, specifically the need to create a physical location for consultations to respect patients' privacy within a pharmacy.

In the course of the project 'Redefinition and approbation of the academic curricula and syllabi of the Faculty of Pharmacy at the Medical University 'Prof. Dr. Paraskev Stoyanov' of Varna in compliance with the needs and requirements of the labor market' within the framework of the European Social Fund, Human Resources Development Operational Programme of the European Commission, Bulgarian pharmacists' attitudes in this respect were comprehensively evaluated (26). The assessment of a representative sample of 100 respondents indicated a relatively highly perceived significance of the education in pharmaceutical care along with a less sufficient practical training in this discipline in Bulgaria.

\section{CONCLUSION}

In recent years, convincing evidence accumulates of the feasibility and effectiveness of a variety of interventions immediately associated with pharmaceutical care aiming at improving patients' relationships with master pharmacists in public pharmacies. Hospital pharmacists recommend a broader practical implementation of pharmaceutical care. The interactive pharmacy students' learning can enhance their pharmaceutical care competences. The collaboration between health authorities and educational institutions along with the integration of innovative approaches in the management of community and hospital pharmacies and pharmacy education is necessary to overcome the organizational, technical and professional barriers in this respect.

\section{REFERENCES}

1. Manasse HR Jr. Medication use in an imperfect world: drug misadventuring as an issue of public policy, Part 1. Am J Hosp Pharm. 1989;46(5):929-44.

2. Manasse HR Jr. Medication use in an imperfect world: drug misadventuring as an issue of public policy, Part 2. Am J Hosp Pharm. 1989a;46(6):1141-52.

3. Hepler CD, Strand LM. Opportunities and responsibilities in pharmaceutical care. Am J Hosp Pharm. 1990;47(3):533-43.

4. Hepler CD. Unresolved issues in the future of pharmacy. Am J Hosp Pharm. 1988;45(5):1071-81.

5. Arranz Alvarez L. Pharmaceutic care: paradigm of health for all in the year 2000? Rev Sanid Hig Publica (Madr). 1990;64(1-2):9-15. Spanish.

6. Penna RP. Pharmaceutical care: pharmacy's mission for the 1990s. Am J Hosp Pharm. 1990;47(3):543-9.

7. Hepler CD. The future of pharmacy: pharmaceutical care. Am Pharm. 1990;NS30(10):23-9.

8. Hepler CD, Strand LM, Tromp D, Sakolchai S. Critically examining pharmaceutical care. J Am Pharm Assoc (Wash). 2002;42(5 Suppl 1):S18-9.

9. Galinsky RE, Nickman NA. Pharmacists and the mandate of pharmaceutical care. DICP. 1991;25(4):431-4.

10. Awalom MT, Tesfa AF, Kidane ME, Ghebremedhin MR, Teklesenbet AH. Eritrean pharmacists' job satisfaction and their attitude to re-professionalize pharmacy in to pharmaceutical care. Int J Clin Pharm. 2015;37(2):335-41.

11. Katoue MG, Awad AI, Schwinghammer TL, Kombian SB. Pharmaceutical care in Kuwait: hospital pharmacists' perspectives. Int J Clin Pharm. 2014;36(6):1170-8.

12. Helling DK, Johnson SG. Defining and advancing ambulatory care pharmacy practice: it is time to lengthen our stride. Am J Health Syst Pharm. 2014;71(16):1348-56.

13. Tommelein E, Tollenaere K, Mehuys E, Boussery K. Pharmaceutical care for patients with COPD in 
Belgium and views on protocol implementation. Int J Clin Pharm. 2014;36(4):697-701.

14. Zhu L, Fox A, Chan YC. Enhancing collaborative pharmaceutical care for patients with chronic kidney disease: survey of community pharmacists. Can J Hosp Pharm. 2014;67(4):268-73.

15. Prot-Labarthe S, Lavoie A, Bourdon O, Lebel D, Bussières JF, Faye A, et al. Étude pilote sur la mise en place des soins pharmaceutiques dans le service de pédiatrie générale d'un hôpital français. Collaboration et comparaison franco-québécoise. Arch Pediatr. 2007;14(4):345-53.

16. Kuhmmer R, Lima KM, Ribeiro RA, Hammes LS, Bastos GA, Cotta de Souza MC, et al. Effectiveness of pharmaceutical care at discharge in the emergency department: study protocol of a randomized controlled trial. Trials. 2015;16: 60. doi: 10.1186/ s13063-015-0579-3.

17. Farley TM, Shelsky C, Powell S, Farris KB, Carter BL. Effect of clinical pharmacist intervention on medication discrepancies following hospital discharge. Int J Clin Pharm. 2014;36(2):430-7.

18. Geurts MM, Zuydgeest IA, Walser S, Kijlstra NB, Petkova V, de Gier JJ. The evaluation of patient selfcompletion concordance forms used in community pharmacy: a comparison of two European countries. Int J Pharm Pract. 2014;22(3):186-92.

19. Losada-Camacho M, Guerrero-Pabon MF, Garcia-Delgado P, Martínez-Martinez F. Impact of a pharmaceutical care programme on health-related quality of life among women with epilepsy: a randomised controlled trial (IPHIWWE study). Health Qual Life Outcomes. 2014;12: 162. doi: 10.1186/s12955-014-0162-8.

20. Joost R, Dörje F, Schwitulla J, Eckardt KU, Hugo C. Intensified pharmaceutical care is improving immunosuppressive medication adherence in kidney transplant recipients during the first post-transplant year: a quasi-experimental study. Nephrol Dial Transplant. 2014;29(8):1597-607.

21. Wei L, Yang X, Li J, Liu L, Luo H, Zheng Z, Wei Y. Effect of pharmaceutical care on medication adherence and hospital admission in patients with chronic obstructive pulmonary disease (COPD): a randomized controlled study. J Thorac Dis. 2014;6(6):656-62.

22. Farris KB, Carter BL, Xu Y, Dawson JD, Shelsky C, Weetman DB, et al. Effect of a care transition inter- vention by pharmacists: an RCT. BMC Health Serv Res. 201418;14: 406. doi: 10.1186/1472-6963-14-406.

23. Mesquita AR, Souza WM, Boaventura TC, Barros IM, Antoniolli AR, Silva WB, et al. The effect of active learning methodologies on the teaching of pharmaceutical care in a Brazilian pharmacy faculty. PLoS One. 2015;10(5):e0123141.

24. Katoue MG, Awad AI, Schwinghammer TL, Kombian SB. Pharmaceutical care education in Kuwait: pharmacy students' perspectives. Pharm Pract (Granada). 2014;12(3):411.

25. Perraudin C, Brion F, Bourdon O, Pelletier-Fleury N. The future of pharmaceutical care in France: a survey of final-year pharmacy students' opinions. BMC Clin Pharmacol. 2011;11: 6. doi: 10.1186/1472-6904-11-6.

26. Naydenov T, Pavlov D, Ivanova D, Kerekovska A, Getov I. Relevance of pharmaceutical higher education to the labor market: a study of pharmacists' attitudes. Scripta Scientifica Pharmaceutica. 2014;1(2):7-17. 